\title{
Enhanced Shot Noise in Tunneling through a Stack of Coupled Quantum Dots
}

\author{
P. Barthold, ${ }^{1, *}$ F. Hohls, ${ }^{1,2}$ N. Maire, ${ }^{1}$ K. Pierz, ${ }^{3}$ and R. J. Haug ${ }^{1}$ \\ ${ }^{1}$ Institut für Festkörperphysik, Universität Hannover, Appelstrasse 2, D-30167 Hannover, Germany \\ ${ }^{2}$ Cavendish Laboratory, University of Cambridge, Madingley Road, Cambridge CB3 OHE, United Kingdom \\ ${ }^{3}$ Physikalisch-Technische Bundesanstalt, Bundesallee 100, D-38116 Braunschweig, Germany
}

(Received 9 February 2006; published 21 June 2006)

\begin{abstract}
We have investigated the noise properties of the tunneling current through vertically coupled selfassembled InAs quantum dots. We observe super-Poissonian shot noise at low temperatures. For increased temperature this effect is suppressed. The super-Poissonian noise is explained by capacitive coupling between different stacks of quantum dots.
\end{abstract}

DOI: $10.1103 /$ PhysRevLett.96.246804

PACS numbers: 73.63.Kv, 72.70.+m, 73.40.Gk

Shot noise was introduced by Walter Schottky in 1918 while looking at the current fluctuations of vacuum tubes [1]. Because of the discreteness of the electrons and the stochastic emission, the current through the device fluctuates around its average value. The corresponding shot noise power is frequency independent and therefore called white noise. A comparable effect is a single tunneling barrier in a semiconductor device. The observed noise power density $S$ follows the same expression $S=2 e I$ where $I$ is the average current and $e$ is the electron charge [2]. It was found that the shot noise power was reduced for tunneling through a double-barrier structure $S<2 e I$. Observations of a reduced noise power density have been made for quantum well structures where the electrons tunnel through a two-dimensional subband $[3,4]$ and for systems with zero-dimensional states [5-8]. This reduction is attributed to a negative correlation between the tunneling events due to the finite dwell time of the resonant state [9-11]. A positive correlation between the individual tunneling events has been observed as well, which leads to an enhanced or so-called super-Poissonian noise power $[12,13]$. The reasons for super-Poissonian shot noise depend on the details of the device structure. In Ref. [12] enhanced shot noise is observed in a quantum well and explained by Coulomb interaction and the shape of the density of states in the well. In Ref. [13] holes and the magnetic field were taken into account to explain super-Poissonian shot noise. It was shown that an impurity which is only weakly coupled to the leads can modulate the tunneling current through a nearby impurity leading to enhanced shot noise [14]. Super-Poissonian noise also has been studied experimentally for triple-barrier resonant-tunneling diodes [15].

In this Letter we study the shot noise properties of resonant tunneling through vertically coupled zerodimensional systems, so-called quantum dots (QDs). For given bias voltages the current flows through a stack of vertical coupled InAs quantum dots [16]. Therefore we deal with $3 d-0 d-0 d-3 d$ tunneling in contrast to the above-mentioned experimental studies where the tunneling takes place through a two-dimensional subband [15].

The QDs were prepared by growing InAs on AlAs, the lattice-mismatch of InAs and AlAs causes the formation of the pyramidal QDs (Stranski-Krastanov growth). The active region of the sample consists of two layers of selfassembled InAs QDs (Fig. 1, see also [16]). The bottom layer contains QDs with a diameter of $10-15 \mathrm{~nm}$ and a height between 2 and $4 \mathrm{~nm}$. The QDs that are formed in the second layer are aligned to the dots in the first layer due to the remaining strain. The QDs in the second layer are slightly larger than the QDs in the first one [17]. These two layers of QDs are surrounded by AlAs barriers. The three-dimensional emitter as well as the collector are given by a $15 \mathrm{~nm}$ undoped GaAs spacer followed by GaAs buffer with graded doping on both sides. The contacts are realized by annealed $\mathrm{Au} / \mathrm{Ge} / \mathrm{Ni} / \mathrm{Au}$ contacts. About $1 \times 10^{6}$ QDs are placed randomly on the area of an etched diode structure of $40 \mu \mathrm{m} \times 40 \mu \mathrm{m}$. It has been shown that in similar samples with one layer of self-assembled QDs only a small
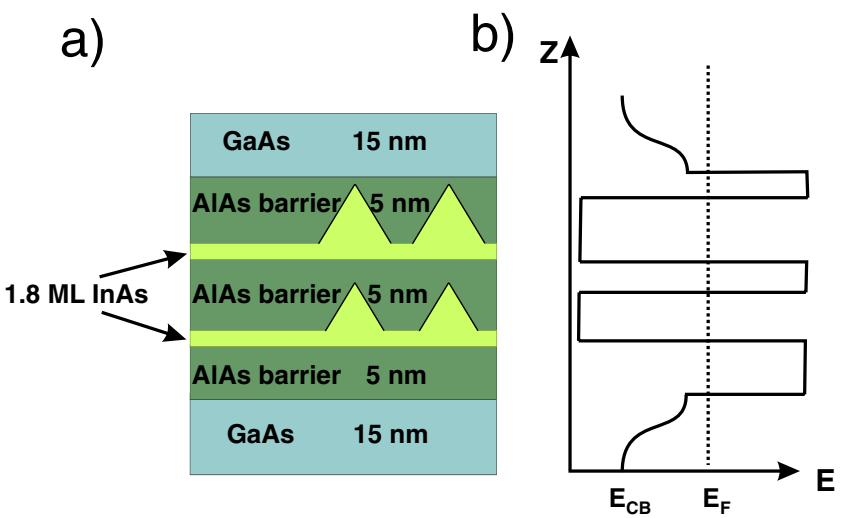

FIG. 1 (color online). (a) Growth scheme of the active part of the device. (b) Conduction band structure $E_{\mathrm{CB}}$ and the Fermi energy $E_{F}$ of a stack of two QDs. If a positive bias voltage is applied the electrons tunnel in positive $z$ direction through the device. 
fraction of these QDs participates in the electronic transport [18]. It seems reasonable that in stacked QDs even less QDs allow electronic transport through the diodes [16].

To investigate the noise properties the sample is placed in a ${ }^{4} \mathrm{He}$ bath cryostat. During the measurements the sample is always immersed in liquid helium. We can control the temperature between 1.4 and $4 \mathrm{~K}$. The external bias voltage $V_{\mathrm{SD}}$ is applied between the source and the drain electrodes using a filtered dc-voltage source. The detected signal is amplified by a low-noise current amplifier with a $3 \mathrm{~dB}$ bandwidth of $10 \mathrm{kHz}$. The signal is fed into a fast Fourier transform analyzer (FFT) to characterize the frequency spectra in a range from 16 to $12.8 \mathrm{kHz}$ with $16 \mathrm{~Hz}$ resolution. In addition we measure the stationary current.

Figure 2(a) demonstrates a part of the current-voltage characteristic of the sample at $T \approx 1.4 \mathrm{~K}$. The curve shows three well-defined peaks. To understand this behavior we take a look at the inset. It shows the schematic band structure of the sample with external voltage $V_{\mathrm{SD}}$ applied. On the left side we see the three-dimensional emitter followed by three barriers. In between these barriers are two quantized states, the ground states of the vertically coupled InAs quantum dots. On the right side the threedimensional collector is shown. If there is no external voltage applied to the sample, the two states of the QDs are above the Fermi energy of the emitter and the collector. By applying an external voltage $V_{\mathrm{SD}}$ we lower the energy levels of the dots and the collector in comparison to the Fermi energy of the emitter. At a certain voltage the two states in the QDs are in resonance. At this point electrons are able to tunnel from the emitter into the first and then into the second QD and from there into unoccupied states
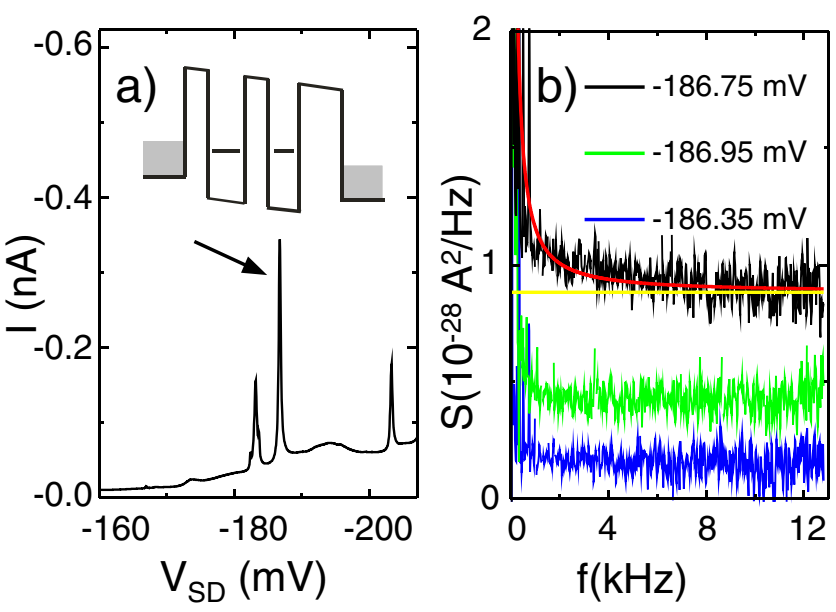

FIG. 2 (color online). (a) Current-voltage characteristics of the sample at $T \approx 1.4 \mathrm{~K}$. The arrow marks the peak which is discussed further. Inset: Schematic band structure of the coupled quantum dots where resonant tunneling is observed. (b) Typical noise spectra of the sample for different bias voltages. The solid line demonstrates the results of fitting the function $A / f+S_{0}$ to the spectrum at $V_{\mathrm{SD}}=-186.75 \mathrm{mV}$. The horizontal line shows $S_{0}$. of the collector. This process of sequential tunneling causes a peak in the current-voltage characteristic. If the states in the QDs are not resonant an electron has to tunnel through the three barriers at once creating a small leakage current. Such a behavior is described in detail in Refs. $[16,19]$.

In Fig. 2(b) we present typical noise spectra of the sample. Spectra as the ones for $V_{\mathrm{SD}}=-186.35 \mathrm{mV}$ and $V_{\mathrm{SD}}=-186.95 \mathrm{mV}$ show a frequency independent behavior above $4 \mathrm{kHz}$. At very low frequencies the spectra show typically a $1 / f$ behavior. We fit the function $A / f+$ $S_{0}$ to the spectra to obtain information about the amplitude of the shot noise $S_{0}$. By comparing $S_{0}$ to the averaged noise spectra between 5 and $12.8 \mathrm{kHz}$ we find that $S_{0}$ provides reliable information.

In Fig. 3 we take a closer look at the marked peak in the current-voltage characteristic [Fig. 2(a)]. The left axis in Fig. 3 demonstrates the current $I$ (dashed line). The right axis shows the noise density $S_{0}$ (line with dots). The squares correspond to the spectra shown in Fig. 2. The scale on the axis is chosen such that the dashed line also corresponds to full shot noise $S_{\text {full }}=2 e I$ on the right axis. Coming from low voltages $V_{\mathrm{SD}}$, the shot noise power $S$ is slightly suppressed. At $V_{\mathrm{SD}} \approx-186.4 \mathrm{mV}$ the shot noise power starts to be larger than the full (Poissonian) shot noise (dashed line). We observe an enhanced shot noise power at both sides of the peak while the shot noise is reduced at the maximum of the current peak. Above $V_{\mathrm{SD}} \approx$ $-186.95 \mathrm{mV}$ the shot noise begins to be suppressed again.

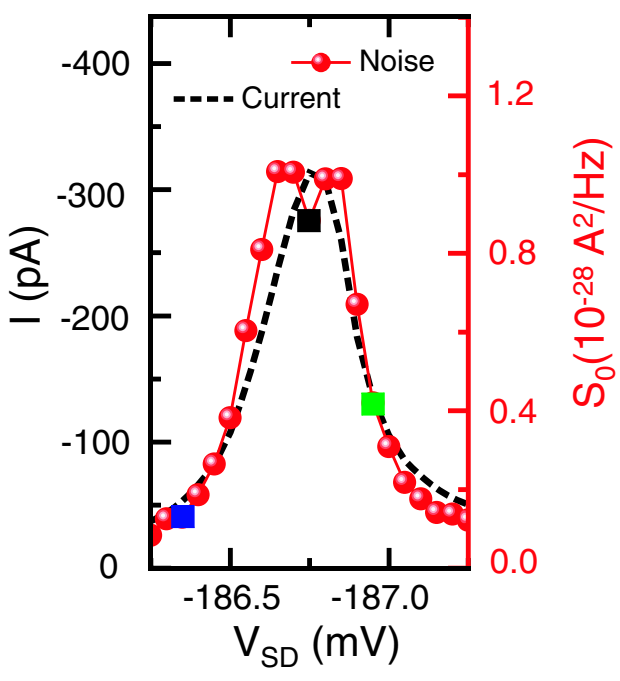

FIG. 3 (color online). Blow up of the peak marked in Fig. 2 in the current-voltage characteristic measured at $T \approx 1.4 \mathrm{~K}$ (dashed line, left axis) and shot noise amplitude $S_{0}$ as given by fitting the function $A / f+S_{0}$ to the noise spectra shown in Fig. 2 (dots, right axis). The squares correspond to the spectra shown in Fig. 2(b). The scale of the right axis was chosen such that the dashed line corresponds on this axis to full shot noise $S_{\text {full }}=2 e I$ as expected for a single tunneling barrier structure (Fano factor $\alpha=1$ ). 


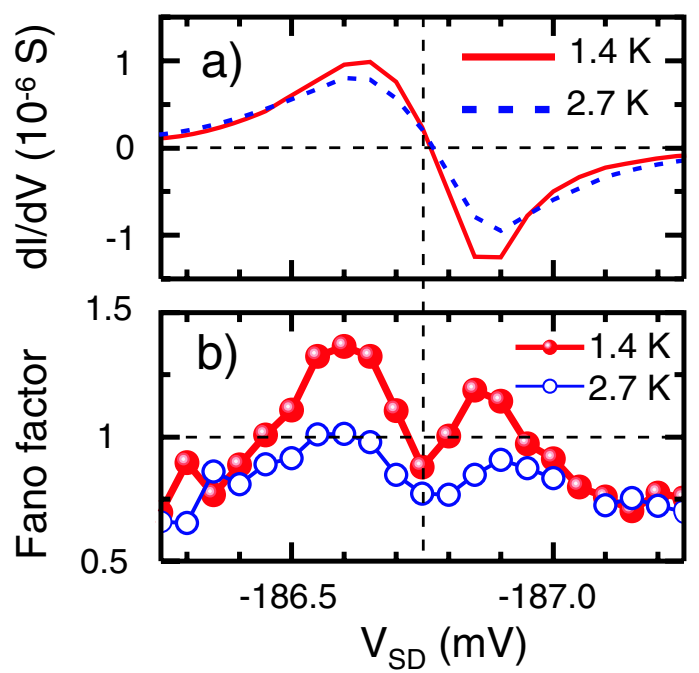

FIG. 4 (color online). (a) Differential conductance for a peak in the $I-V$ characteristic at $T \approx 1.4 \mathrm{~K}$ and $T \approx 2.7 \mathrm{~K}$. (b) The filled symbols show the Fano Factor $\alpha$ at $T \approx 1.4 \mathrm{~K}$ and the open circles show $\alpha$ at $T \approx 2.7 \mathrm{~K}$.

In Fig. 4(a) the differential conductance of the sample is shown for $T \approx 1.4 \mathrm{~K}$ and $T \approx 2.7 \mathrm{~K}$ for the same range of $V_{\mathrm{SD}}$ as used in Fig. 3. To compare the differential conductance with the shot noise amplitude we display the socalled Fano factor $\alpha$ in the figure beneath [Fig. 4(b)]. The Fano factor compares the measured shot noise power $S_{0}$ and the full shot noise $2 e I: \alpha:=S_{0} /(2 e I)$. The filled symbols in Fig. 4(b) show the Fano factor $\alpha$ for $T \approx 1.4 \mathrm{~K}$ while the open symbols show $\alpha$ for $T \approx 2.7 \mathrm{~K}$.

For $T \approx 1.4 \mathrm{~K}$ the Fano factor $\alpha$ displays a distinct double-peak behavior. The peaks in the Fano factor are clearly reduced at $T \approx 2.7 \mathrm{~K}$, however, the double-peak structure is evident for both temperatures. At lower voltages than $V_{\mathrm{SD}} \approx-186.45 \mathrm{mV}$ the Fano factor $\alpha$ is smaller than 1 for both temperatures. While the differential conductance increases the Fano factor rises to values above 1. The maximum in the differential conductance coincides with the first maximum of the Fano factor $\alpha$ at $V_{\mathrm{SD}} \approx$ $-186.65 \mathrm{mV}$ at both temperatures where the values of the Fano factor are $\alpha \approx 1.40$ at $T \approx 1.4 \mathrm{~K}$ and $\alpha \approx 1.03$ at $T=2.7 \mathrm{~K}$. Together with the decreasing differential conductance, the Fano Factor drops to its minimum where the differential conductance is zero. This minimum is nearly independent of the temperature and has a value of about 0.89 for $T \approx 1.4 \mathrm{~K}$ and 0.78 for $T \approx 2.7 \mathrm{~K}$. With further rise of the bias voltage the differential conductance shows its minimum where the Fano factor $\alpha$ reaches its second local maximum at $\alpha \approx 1.22$ at $T \approx 1.4 \mathrm{~K}$ and $\alpha \approx 0.9$ at $T \approx 2.7 \mathrm{~K}$. Comparing the temperature dependence of the Fano factor to the differential conductance we find that the latter shows a much smaller temperature dependence. We also performed noise measurements on stacked InAs dots in InP barriers with GaInAs leads as described in Ref. [19]. These devices showed a similar behavior as presented here.
The observed double-peak structure of super-Poissonian noise is quite astonishing, because theoretical approaches (like Ref. [20]), describing only a pair of vertically coupled QDs, expect a Fano factor $\alpha<1$. In contrast, theoretical models that take coupling mechanisms to other systems into account predict super-Poissonian shot noise.

A Monte Carlo and analytic simulation of four metallic dots arranged as two parallel, only capacitively coupled double dots showed an enhanced Fano factor with a double-peak structure very similar to our experiment [21]. Here the enhancement of the shot noise stems from the Coulomb interaction of neighboring QDs. Each peak in the Fano factor occurs where the difference between both currents through the parallel metallic QDs reaches its maximum. Gattobigio et al. [21] showed that a so-called locking effect occurs due to electrostatic coupling between two pairs of dots. A similar scenario is quite likely in our device as it consists of a large number of stacked selfassembled dots. It is reasonable that two or more stacks might conduct at the same bias voltage.

Gattobigio et al. [21] have shown that the value of the maxima in the Fano factor depends on the ratio between the tunneling resistances of each parallel pair of QDs. A ratio of 1 leads to a symmetric double peak in the Fano factor, other tunneling resistances lead to an asymmetric Fano factor. This could be an explanation for the strong temperature dependence of the Fano factor that is observed. It is possible that this ratio is changed in our device by changing the temperature which would influence the maximum and the ratio of the two peaks in the Fano factor. With rising ratio of the tunneling resistances the asymmetry of the double peak in the Fano factor grows. Supposing that the temperature dependence of the QD which contributes less current is larger than the other, it would be easy to understand why the current peak shows only slight changes with rising temperature while the Fano factor depending on the ratio of the two tunneling resistances shows a strong temperature dependence.

Another coupling between two systems that causes an enhancement in the shot noise was reported in Ref. [22]. Koch et al. [22] have shown that the Fano factor can rise to giant values in transport through a single molecule where a strong coupling between phonons and electrons exists. This calculations were done in the Franck-Condonblockade regime. It is also possible that such a phononelectron coupling might play a role in our device.

For a quantitative description of the observed shot noise, further detailed calculations for such a device are necessary.

In conclusion, we presented measurements of shot noise on self-assembled coupled InAs QDs. To characterize the shot noise we introduced the Fano factor $\alpha$. The Fano factor $\alpha$ shows a double-peak structure with maxima above 1, i.e., super-Poissonian noise which is explained in terms of coupling between different stacks of quantum dots. 
We acknowledge financial support from BMBF.

*Electronic address: Barthold@nano.uni-hannover.de

[1] W. Schottky, Ann. Phys. (Leipzig) 57, 541 (1918).

[2] Ya. M. Blanter and M. Büttiker, Phys. Rep. 336, 1 (2000).

[3] Y.P. Li, A. Zaslavsky, D.C. Tsui, M. Santos, and M. Shayegan, Phys. Rev. B 41, 8388 (1990).

[4] H. C. Liu, J. Li, G. C. Aers, C. R. Leavens, M. Buchanan, and Z. R. Wasilewski, Phys. Rev. B 51, 5116 (1995).

[5] H. Birk, M. J. M. de Jong, and C. Schönenberger, Phys. Rev. Lett. 75, 1610 (1995).

[6] A. Nauen, I. Hapke-Wurst, F. Hohls, U. Zeitler, R. J. Haug, and K. Pierz, Phys. Rev. B 66, 161303(R) (2002).

[7] A. Nauen, F. Hohls, J. Könemann, and R. J. Haug, Phys. Rev. B 69, 113316(R) (2004).

[8] A. Nauen, F. Hohls, N. Maire, K. Pierz, and R. J. Haug, Phys. Rev. B 70, 033305(R) (2004).

[9] L. Y. Chen and C.S. Ting, Phys. Rev. B 43, 4534 (1991).

[10] J.H. Davies, P. Hyldgaard, S. Hershfield, and J. W. Wilkins, Phys. Rev. B 46, 9620 (1992).

[11] G. Kießlich, A. Wacker, E. Schöll, A. Nauen, F. Hohls, and R. J. Haug, Phys. Stat. Solidi C 0, No. 4, 1293 (2003).
[12] G. Iannaccone, G. Lombardi, M. Macucci, and B. Pellegrini, Phys. Rev. Lett. 80, 1054 (1998).

[13] V. V. Kuznetsov, E. E. Mendez, J.D. Bruno, and J.T. Pham, Phys. Rev. B 58, R10 159 (1998).

[14] S. S. Safonov, A. K. Savchenko, D. A. Bagrets, O. N. Jouravlev, Y. V. Nazarov, E. H. Linfield, and D. A. Ritchie, Phys. Rev. Lett. 91, 136801 (2003).

[15] A. K. M. Newaz, W. Song, E. E. Mendez, Y. Lin, and J. Nitta, Phys. Rev. B 71, 195303(R) (2005).

[16] D. Sarkar, U. Zeitler, I. Hapke-Wurst, R. J. Haug, and K. Pierz, in Proceedings of the 26th International Conference on the Physics of Semiconductors, Edinburgh, 2002, IOP Conference Series 171, edited by A. R. Long and J. H. Davies (IOP, Bristol, 2003), p. 233.

[17] H. Eisele, O. Flebbe, T. Kalka, C. Preinesberger, F. Heinrichsdorff, A. Krost, D. Bimberg, and M. DähnePietsch, Appl. Phys. Lett. 75, 106 (1999).

[18] I. Hapke-Wurst, U. Zeitler, U.F. Keyser, R. J. Haug, K. Pierz, and Z. Ma, Appl. Phys. Lett. 82, 1209 (2003).

[19] T. Bryllert, M. Borgstrom, L.-E. Wernersson, W. Seifert, and L. Samuelson, Appl. Phys. Lett. 82, 2655 (2003).

[20] G. Kießlich, P. Samuelsson, A. Wacker, and E. Schöll, Phys. Rev. B 73, 033312 (2006).

[21] M. Gattobigio, G. Iannaccone, and M. Macucci, Phys. Rev. B 65, 115337 (2002).

[22] J. Koch and F. von Oppen, Phys. Rev. Lett. 94, 206804 (2005). 